NASA/TM-2002-211907

Validation of WIND for a Series of Inlet Flows

John W. Slater, John M. Abbott, and Richard H. Cavicchi

Glenn Research Center, Cleveland, Ohio 
Since its founding, NASA has been dedicated to the advancement of aeronautics and space science. The NASA Scientific and Technical Information (STI) Program Office plays a key part in helping NASA maintain this important role.

The NASA STI Program Office is operated by Langley Research Center, the Lead Center for NASA's scientific and technical information. The NASA STI Program Office provides access to the NASA STI Database, the largest collection of aeronautical and space science STI in the world. The Program Office is also NASA's institutional mechanism for disseminating the results of its research and development activities. These results are published by NASA in the NASA STI Report Series, which includes the following report types:

- $\quad$ TECHNICAL PUBLICATION. Reports of completed research or a major significant phase of research that present the results of NASA programs and include extensive data or theoretical analysis. Includes compilations of significant scientific and technical data and information deemed to be of continuing reference value. NASA's counterpart of peerreviewed formal professional papers but has less stringent limitations on manuscript length and extent of graphic presentations.

- TECHNICAL MEMORANDUM. Scientific and technical findings that are preliminary or of specialized interest, e.g., quick release reports, working papers, and bibliographies that contain minimal annotation. Does not contain extensive analysis.

- CONTRACTOR REPORT. Scientific and technical findings by NASA-sponsored contractors and grantees.
- CONFERENCE PUBLICATION. Collected papers from scientific and technical conferences, symposia, seminars, or other meetings sponsored or cosponsored by NASA.

- SPECIAL PUBLICATION. Scientific, technical, or historical information from NASA programs, projects, and missions, often concerned with subjects having substantial public interest.

- TECHNICAL TRANSLATION. Englishlanguage translations of foreign scientific and technical material pertinent to NASA's mission.

Specialized services that complement the STI Program Office's diverse offerings include creating custom thesauri, building customized databases, organizing and publishing research results ... even providing videos.

For more information about the NASA STI Program Office, see the following:

- Access the NASA STI Program Home Page at http://www.sti.nasa.gov

- E-mail your question via the Internet to help@sti.nasa.gov

- Fax your question to the NASA Access Help Desk at 301-621-0134

- Telephone the NASA Access Help Desk at 301-621-0390

- Write to:

NASA Access Help Desk

NASA Center for AeroSpace Information 7121 Standard Drive

Hanover, MD 21076 
NASA/TM-2002-211907

AIAA-2002-0669

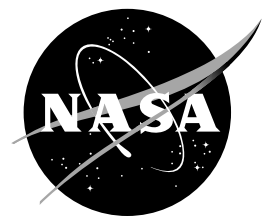

\section{Validation of WIND for a Series of Inlet Flows}

John W. Slater, John M. Abbott, and Richard H. Cavicchi

Glenn Research Center, Cleveland, Ohio

Prepared for the

40th Aerospace Sciences Meeting and Exhibit

sponsored by the American Institute of Aeronautics and Astronautics

Reno, Nevada, January 14-17, 2002

National Aeronautics and

Space Administration

Glenn Research Center 


\section{Acknowledgments}

The authors would like to thank Dave Saunders, Rene Fernandez, Dr. Jim DeBonis, and Dr. Charles Trefny of the NASA Glenn Research Center for assistance with the experimental data.

The Aerospace Propulsion and Power Program at NASA Glenn Research Center sponsored this work.

Available from

NASA Center for Aerospace Information 7121 Standard Drive

Hanover, MD 21076
National Technical Information Service 5285 Port Royal Road Springfield, VA 22100

Available electronically at http:/ /gltrs.grc.nasa.gov 


\title{
Validation of WIND for a Series of Inlet Flows
}

\author{
John W. Slater, ${ }^{*}$ John. M. Abbott, ${ }^{\dagger}$ and Richard H. Cavicchi ${ }^{\ddagger}$ \\ National Aeronautics and Space Administration \\ Glenn Research Center \\ Cleveland, Ohio 44135
}

\begin{abstract}
$\underline{\text { Abstract }}$
Validation assessments compare WIND CFD simulations to experimental data for a series of inlet flows ranging in Mach number from low subsonic to hypersonic. The validation procedures follow the guidelines of the AIAA. The WIND code performs well in matching the available experimental data. The assessments demonstrate the use of WIND and provide confidence in its use for the analysis of aircraft inlets.
\end{abstract}

\section{$\underline{\text { Introduction }}$}

The NASA Glenn Research Center (GRC) is involved in the design and analysis of inlet concepts for aerospace vehicles that operate at Mach numbers spanning the range from low subsonic to hypersonic. Inlet flow fields are fairly complex. The external flow enters the inlet and passes through internal ducting that may involve significant turning and curvature to reach the engine. The curvature of internal ducting promotes secondary flows. The viscous flow on the vehicle and inlet surfaces may consist of laminar boundary layers transitioning to turbulent boundary layers. The inlet is a flow compression device, and so, the flow exists in an adverse pressure gradient. Supersonic flows usually contain shock waves that then interact with the boundary layers. All these features potentially lead to boundary layer separation. At hypersonic Mach numbers, the possibility of high-temperature effects exists. The flow may consist of local unsteady features such as time-varying shocks, boundary layers, or boundary layer separation regions. There may exist in the inlet flow control devices such as bleed holes and slots, jets, and vortex generators.

An analysis of the flow in an inlet should provide qualitative insight into the general flow features, such

\footnotetext{
* Aerospace Engineer, AIAA Senior Member.

$\dagger$ Senior Researcher, AIAA Member

$\ddagger$ Aerospace Engineer
}

as the character of the boundary layers and the location of shocks. An analysis should also provide values for the measures of performance of the inlet. The primary measure of performance is the total pressure recovery, which is defined as the ratio of an average total pressure at the inlet exit to the freestream total pressure. It represents the amount of loss of energy in the flow due to viscosity and shock waves. At hypersonic speeds, the kinetic energy efficiency is often used as the measure of performance. Other flow properties to be determined include the mass flow through the inlet and the distribution of pressures on the inlet surfaces.

Computational fluid dynamics (CFD) is a useful tool for performing the aerodynamic analysis of the inlet flows. The CFD analysis process first involves defining the flow problem and establishing what results are required from the analysis. The inlet geometry and flow domain are modeled and the grid is generated. The boundary and initial conditions are then specified and the CFD code is then run to obtain the flow field. The flow field is then analyzed to obtain the performance measures of the inlet. Of primary concern is having confidence in the CFD methods for providing accurate, credible results.

Credibility is obtained by performing verification and validation assessments. Here verification and validation are defined according to the AIAA guideline. ${ }^{1}$ Verification is defined as the process of determining that a model implementation accurately represents the developer's conceptual description of the model and the solution to the model. Verification assessment looks for errors in the numerical approximation of the models and the programming of the code. Such errors are uncovered through careful visual and computational examination of the code and through comparison of CFD simulation results to highly accurate solutions to model problems. Validation is defined as the process of determining the degree to which a model is an accurate representation of the real world from the perspective of the intended uses of the model. Validation assessment looks for errors in the 
geometric and physical models through comparison of the CFD simulation results to experimental data, which is assumed to be the controlled observation of the real world. Both verification and validation assessment require that the solutions are consistent with physical models and that the levels of iterative convergence error and spatial and temporal discretization errors be examined and quantified. Verification assessment should usually precede validation assessment; however, both are a continuous activity as long as CFD codes incorporate new models and methods and their range of application is extended.

In this paper, the WIND CFD code is assessed. ${ }^{2,3} \mathrm{~A}$ verification assessment of the WIND code for several aspects of inlet flows has been examined in an earlier paper. ${ }^{4}$ The focus of the current paper is the validation assessment of WIND for use in the analysis of inlet flow fields over a Mach number range of 0.1 to 6.0. The next section describes the WIND code. The section following discusses procedures for performing a validation assessment. The remaining sections then discuss validation assessments performed for simulations of five inlet flow fields: the Stanitz elbow, scarf subsonic inlet, the NASA VDC supersonic inlet, the NASA strut-jet hypersonic inlet, and the NASA GTX hypersonic inlet.

\section{WIND CFD Code}

The WIND CFD code is being developed by the NPARC Alliance (National Program for Applicationsoriented Research in CFD), which is an alliance of the NASA Glenn Research Center, the Air Force's Arnold Engineering Development Center, and the Boeing Company. ${ }^{2,3}$ WIND solves the time-dependent, Reynolds-averaged Navier-Stokes equations for turbulent, compressible flows using a cell-vertex, finitevolume, time-marching approach on multi-zone, structured grids. Spatial accuracy is formally secondorder using the Roe flux-difference splitting upwind formulation. Steady flows are simulated through an iterative process using local time stepping. Unsteady flows are simulated through a second-order marching in time; however, the Reynolds-averaging precludes the simulation of anything other than gross unsteady flow features. Turbulence is modeled using algebraic, oneequation, or two-equation eddy viscosity models. The Baldwin-Lomax model is the primary algebraic model. The Spalart-Allmaras model is the primary oneequation model. The SST model is the primary twoequation model, but the Chien $\mathrm{k}-\varepsilon$ model is also available. WIND is capable of solving for flows of speeds ranging from low subsonic to hypersonic. At hypersonic Mach numbers, WIND has models to simulate high-temperature effects in air.

\section{$\underline{\text { Validation Assessment }}$}

The validation assessment of WIND for the analysis of inlet flows recognizes the "building-block" approach suggested in the AIAA Guideline. "The approach involves dividing a complex system such as an inlet into progressively simpler phases: subsystem cases, benchmark cases, and unit problems. Each phase involves simpler level of geometrical complexity and flow physics coupling, as well as, increased levels of experimental accuracy. The validation assessments involve a series of procedures that are discussed in the following paragraphs:

Examine the simulation for iterative convergence. The CFD simulations discussed here are steady-state flow simulations in which the time-marching numerical methods are iterated in pseudo-time until flow properties no longer change. One measure of iterative convergence used for these simulations was the decrease and asymptotic convergence of the residuals of the flow and turbulence equations. Another measure used was the asymptotic convergence of the total pressure recovery and mass flow through the inlet. The typical iteration convergence criterion was that the change in the total pressure recovery be less than 0.00001 over 500 iterations. The amount of variation that is accepted as the convergence criterion is a measure of the iterative convergence error.

Examine the simulation for consistency. The simulations should be consistent with the general principles of the models. For the inlet simulations discussed here the conservation of mass through the duct was checked. Furthermore, the total pressure recovery was checked to see if it decreased, or at least remained constant along the duct.

Examine the simulation for spatial (grid) convergence. The basis of CFD is that as the grid is refined for a steady-state simulation, the CFD results should asymptotically approach the true solution of the equations. For the inlet simulations discussed here, the variation of the static pressure distributions and the total pressure recovery with grid refinement was examined as an indicator of grid convergence. When possible, a grid convergence index (GCI) was calculated as a way of reporting the grid convergence..$^{5}$ The change in pressure recovery with grid refinement indicates the level of spatial discretization error.

Compare the CFD simulation results to the experimental data. The comparison of the CFD results to experimental data examines how well the CFD analysis represents the real world as observed by the experiment. The limits of the experimental data define the limits of the validation assessments. For the inlets considered here, the experimental data consists mainly of static pressure distributions. Thus, the validation assessments primarily assess how well the WIND code 
can predict the static pressure distributions. Any errors in the experimental data are also reported.

Examine the simulation for uncertainties in the physical models. For the inlet cases examined here, the primary models that create the most uncertainty are those modeling turbulence. Simulations were run with at least two turbulence models to examine the level of physical modeling error in the turbulence models.

\section{NPARC Verification and Validation Web Site}

Further details on performing CFD verification and validation assessments can be found on the NPARC Alliance CFD Verification and Validation web site. The web site was created to aid the development and support of the WIND CFD code; however, it is publicly available and provides a useful resource for users of other CFD codes, as well as, the worldwide CFD community. The URL for the web site is http://www.grc.nasa.gov/www/wind/valid. The site includes a fairly self-contained tutorial that includes information on conducting verification and validation assessments. The tutorial also contains a glossary of terms, bibliography, and links to other web sites. The site contains an archive of verification and validation cases representing various geometries and flow conditions. Each case contains information on its geometry, grid, and available analytical and experimental data. Many of the files (grid, solution, input, and output) can be viewed and downloaded. The archive also includes further information on the inlet cases reported in this paper.

\section{$\underline{\text { Inlet Validation Cases }}$}

Validation assessments of the WIND CFD code have been performed for a series of inlet flow cases that span the Mach number range from incompressible through hypersonic speeds. The cases were all experiments conducted at the NASA Glenn Research Center (or one of its predecessors), which in most cases allowed direct access to the people who collected the data. However, the experiments were primarily tests to examine some concept related to inlet flows rather than explicit experiments conducted to provide data for CFD validation assessment. The experimental data mostly consists of static pressure distributions along the surfaces of the inlets. In some of the cases, data for the total pressure recovery or kinetic energy coefficient is reported. All the cases involve steady flow which is mostly turbulent and which high-temperature effects are not considered significant (i.e. calorically perfect air). The following sections discuss each of the cases along with the results of the validation assessments.

\section{$\underline{\text { Stanitz Elbow }}$}

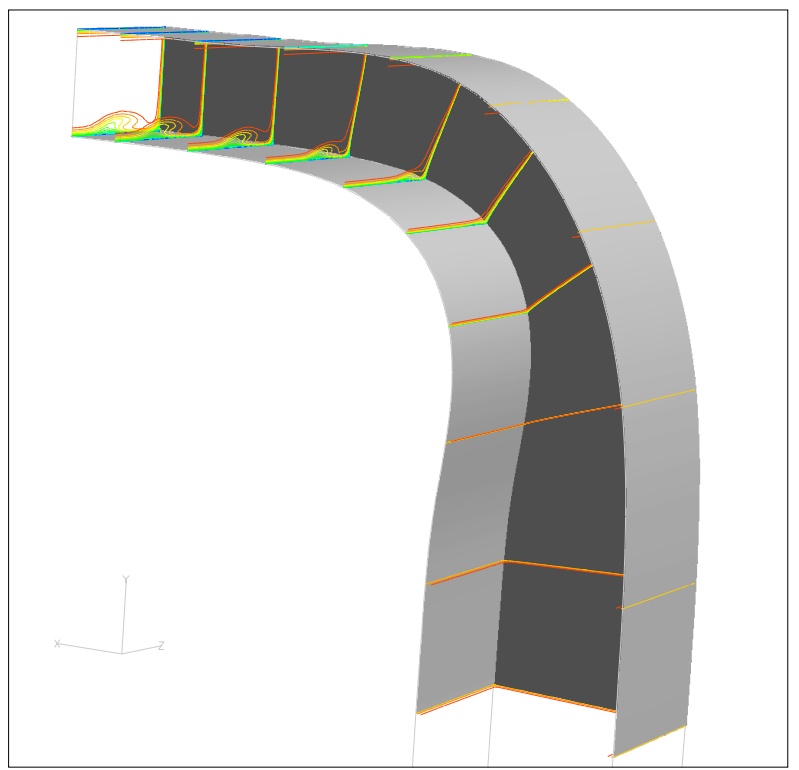

Figure 1. Geometry of the Stanitz elbow with total pressure contours (flow enters from the bottom).

The Stanitz elbow was a duct of rectangular crosssection that was studied at NASA GRC in the early 1950s (at that time the NACA Lewis Flight Propulsion Laboratory) to examine secondary flow structures in low-speed flow ducts with turning. This duct was of interest for a CFD validation study because it represented a class of low-speed internal flows with simple geometry and flow features.

Figure 1 shows the shape of the surfaces of the elbow. The streamwise cross-section was rectangular. The inner (suction) and outer (pressure) surfaces turned the flow approximately 90 degrees.

The flow entered the elbow from the bottom in Fig. 1. The flow was subsonic throughout the elbow with a maximum Mach number of approximately 0.3 , which resulted in the flow being fairly incompressible. The Stanitz elbow was designed using potential flow methods to continually accelerate the flow as is turned through the elbow while avoiding boundary layer separation. The absence of boundary layer separation allowed better examination of the secondary flow features. As the flow accelerated through the elbow, a passage vortex formed on the inner surface and was located nearer the plane sidewall than toward the midspan of the elbow. The sense of the passage vortex was clockwise from the perspective of an observer looking out the exit in the direction of the main flow. Thus the secondary flow imposed a sideways velocity component from the plane sidewall towards the midspan of the elbow. 
The experiment was conducted with the elbow attached to the top of a plenum chamber. The flow went through the elbow and exited into the test facility room. The experiment was conducted with a series of spoilers upstream of the elbow that modified the shape of the boundary layer of the flow entering the elbow. The case considered here is that with no spoiler. The tank gage pressure was 20 inches of water. The mass flow through the elbow was measured from a total pressure rake survey at the exit of the elbow to be $14.60 \mathrm{lbm} / \mathrm{sec}$. The experimental data consist of static pressure distributions on the suction and pressure surfaces at several spanwise stations and total pressure surveys at the exit plane. This case can almost be considered a benchmark validation case in that the geometry and flow are fairly simple; however, the experimental data lacks information on errors.

The geometry was simple and could be modeled fairly accurately. Geometric symmetry along the midplane allowed the modeling of only half of the elbow for the flow domain. The flow domain included the constant-area "tunnel" section that connected the elbow to the plenum chamber. The tunnel section in the experiment had a length of 24 inches. Rather than model the details of the plenum chamber, the inflow boundary was placed at the start of the tunnel section. To match the experimental boundary layer velocity profile at the entrance to the elbow, the length of the tunnel section as modeled in the flow domain was increased to 30 inches. The outflow boundary was placed at the exit of the elbow.

The inflow boundary conditions assumed a total pressure equal to the tank static pressure and a total temperature equal to the ambient static temperature. The outflow boundary condition was specified as a constant mass flow condition set to the mass flow measured in the experiment. The elbow surfaces were assumed to be no-slip, adiabatic walls. A reflection boundary condition was applied at the plane of symmetry of the flow domain. Thus, the boundary conditions were accurately modeled in the CFD simulations. The initial conditions were the plenum conditions with a Mach number of 0.1 assumed.

The rectangular cross-section of the flow domain allowed a highly accurate, single-zone, structured grid to be generated. The grid had 161 grid points in the streamwise direction, 157 grid points from the suction to pressure surface, and 81 grid points from the symmetry plane to the plane wall. The total number of grid points was 2047437. This grid size allowed a medium grid $(81 \times 79 \times 41)$ to be extracted by removing every other grid point in each direction. Similarly a coarse grid $(41 \times 40 \times 21)$ could be extracted by removing every other grid point from the medium grid.

Iterative convergence was determined through examination of the decrease and asymptotic nature of the residual of the flow equations. Further, the pressure recovery at the elbow exit was monitored until absolute changes in the recovery were on the order of 0.00001 over 500 iterations with a clear stabilization of the pressure recovery.

Examining the conservation of mass through the elbow checked the consistency of the solution. The simulations on the fine grid indicated that the mass fluxes integrated on the streamwise grid planes differed by less than $0.1 \%$ of the average mass flow. The differences on the medium grid were about $0.2 \%$.

The spatial or grid convergence was examined by comparing the variation of the total pressure recovery at the elbow exit as determined from simulations on the fine, medium, and coarse grids. Figure 2 shows those variations for simulations performed on the various grids and turbulence models. The relative grid spacing is with respect to the spacing on the fine grid. Thus the medium grid has a relative grid spacing of 2 and the coarse grid has a relative grid spacing of 4 . It should first be noted that the low-speed character of this flow results in very small viscous losses, and so, high recoveries. The recovery for the inviscid simulations should be 1.0 since viscous losses are not to exist. The simulation on the medium grid suggests an error of $0.063 \%$. The trend appears encouraging that, as the grid is refined, the error would reduce further. The laminar simulations indicate slightly more viscous losses; however, the drastic change in slope between the medium and fine grids indicates some irregular behavior in the simulation. The lower recoveries of the turbulence models seem consistent; however, the recoveries from the simulations using the Chien $\mathrm{k}-\varepsilon$ model seem inconsistent with the consistent grouping of the recoveries of the simulations using the Baldwin-Lomax, Spalart-Allmaras, and SST turbulence models. This clearly warrants a closer examination of the $\mathrm{k}-\varepsilon$ model.

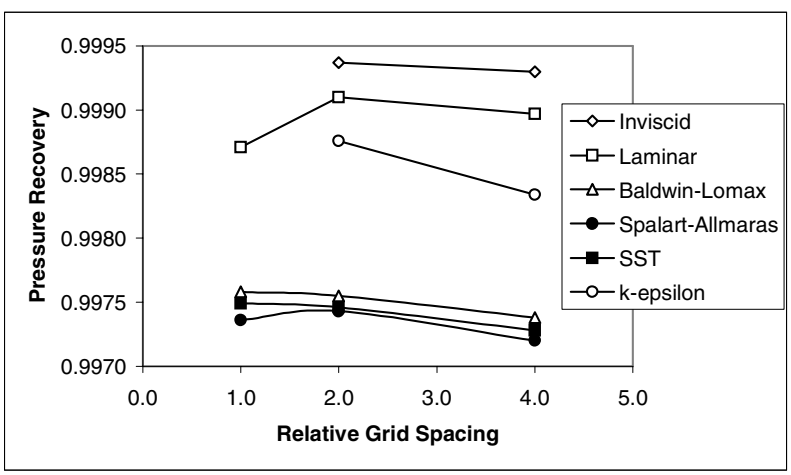

Figure 2. Pressure recovery with respect to grid spacing and turbulence model.

The grid convergence indices (CGI) computed for the variations from the simulations using the BaldwinLomax and SST models were approximately $0.001 \%$ 
and $0.005 \%$ for the fine and coarse grids, respectively. The order of convergence was approximately 2.5 . These results indicated a very small variation of the pressure recovery with grid spacing.

The elbow was designed using potential flow methods such that the exit velocity would be approximately twice that of the inflow velocity. This could be verified through comparison of the theoretical velocity variation through the elbow to that obtained from an inviscid flow simulation. Figure 3 shows that comparison. The velocity plotted for the CFD simulation is an average velocity at each streamwise grid plane. The comparison is very good until towards the exit of the elbow. At the exit, the error is approximately $4 \%$, which is fairly significant and deserves further examination of WIND.

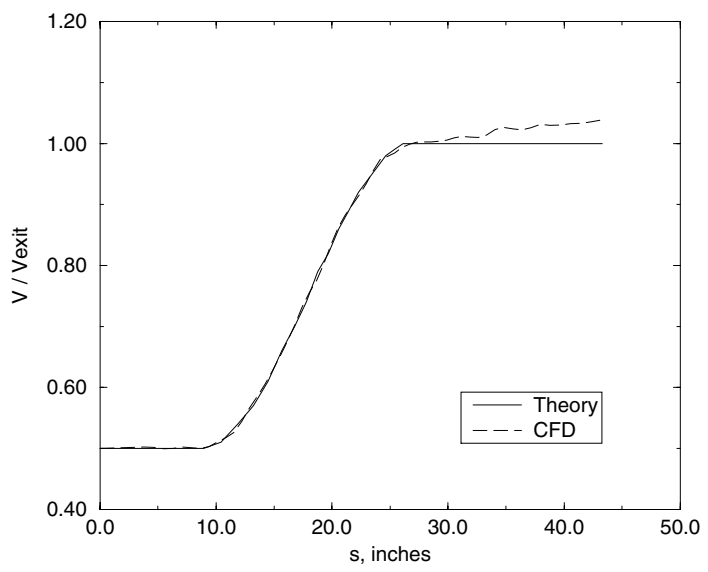

Figure 3. Comparison of the theoretical velocity through the elbow to the average velocity of the inviscid CFD simulation.

Figures 4 and 5 present the comparison of experimental and CFD static pressures on the inner and outer surfaces. Figure 4 presents the streamwise static pressures along the centerline of the elbow for both the inner and outer surfaces. Figure 5 presents the spanwise static pressures at three streamwise stations denoted by the coordinate $\varphi=(0.0,3.0,4.5)$, which relates to the velocity potential used in designing the surfaces of the elbow and scales according to the streamwise distance along the surfaces of the elbow. The experiment report (reference 6) did not comment on the level of error in the experiment, and so, error bars could not be plotted. Figure 4 shows good agreement with the experimental data, but the differences are greatest on the inner surface near the exit. This is the region where the secondary flows are most significant. The absolute difference between the experimental data and the CFD result at the last streamwise data point is approximately
0.03 psi, which is $4.2 \%$ of the tank gage pressure. Figure 4 shows that the static pressures from the simulations on the fine grid were not much different from those on the coarse grid. Furthermore, the inviscid flow simulation indicated that viscosity did not have a huge effect on the static pressures.

Figure 5 shows the spanwise character of the static pressures and shows good agreement of the CFD simulations with the experimental data. The coordinate $\mathrm{z} / \mathrm{w}=0$ indicates the sidewall surface. The increase of static pressure at the sidewall reflects the presence of the passage vortex.

Further discussion of CFD simulations of the Stanitz elbow can be found on the NPARC Verification and Validation web site and in Ref. 7.

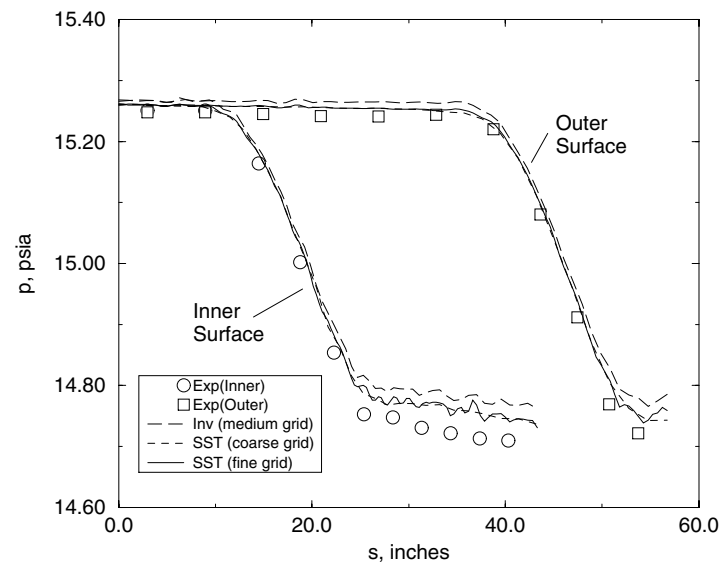

Figure 4. Streamwise distribution of the static pressures on the inner (suction) and outer (pressure) surfaces along the centerline of the elbow.

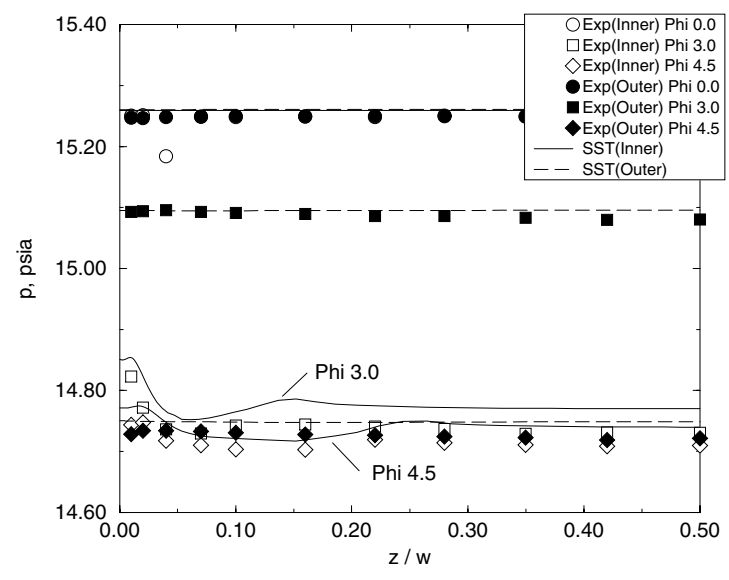

Figure 5. Spanwise distribution of the static pressures on the inner (suction) and outer (pressure) surfaces at streamwise stations $\varphi=(0.0,3.0,4.5)$ of the elbow. 


\section{$\underline{\text { Scarf Subsonic Inlet }}$}

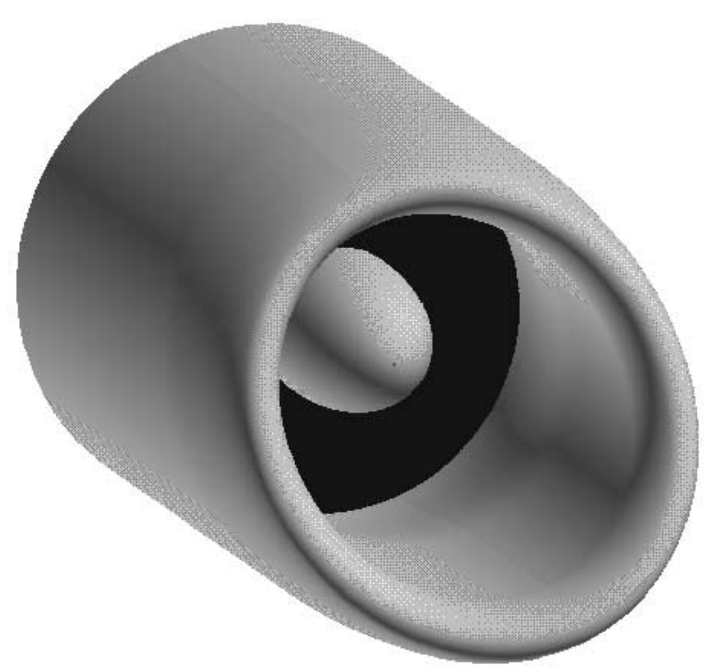

Figure 6. Geometry of the scarf subsonic inlet.

The scarf subsonic inlet was an inlet concept studied at NASA GRC in the late 1970's (at that time the NASA Lewis Research Center) with potential applications for subsonic aircraft. ${ }^{8}$ This inlet concept was studied because it promised lower fly-over noise, less ingestion of foreign objects from the ground during take-off and landing, and greater angle-of-attack tolerance than an axisymmetric inlet with the same lip thickness. This inlet was of interest for a CFD validation study because such three-dimensional subsonic inlets are currently being studied once again at NASA GRC as part of a comprehensive parametric study using the WIND code for the flow analysis. ${ }^{9}$ This validation case directly supported that study.

Figure 6 shows the surfaces of the scarf inlet. The main geometric feature is the lower lip that extends ahead of the upper lip with a straight side profile.

The experimental data available for validation come from tests of the scarf inlet conducted in 1977 in the NASA GRC 9x15-foot wind tunnel. ${ }^{8}$ The inlet was attached to a vacuum system that allowed accurate measurement of the inlet mass flow. The freestream condition of the test was a Mach number of 0.12 with an ambient static pressure of 14.63 psi and static temperature of $533.74{ }^{\circ} \mathrm{R}$. The freestream flow was accelerated into the inlet and reached its highest velocity and lowest pressure as it was turned into the inlet by the inlet lip. The average throat Mach number was 0.63; however, a small transonic region was formed in which the Mach number reached approximately 1.3. A weak normal shock changed the local supersonic flow to subsonic conditions. Aft of the inlet throat, the flow was diffused to decelerate and compress the flow prior to ingestion into the vacuum system.
The experimental data consist of static pressure distributions along the axial direction at 0,90 , and 180-degree stations along the circumference of the inlet and total pressure contours and rake-averaged total pressure recoveries at the exit of the diffuser. The data of interest for this case was collected at 0 and 50 degrees angle-of-attack. This case can be considered a subsystem validation case since the inlet was not attached to an actual engine, and so, considered only the aerodynamics of the flow through the inlet.

The flow domain for the CFD simulations consisted of only one-half of the inlet due to geometric and flowfield symmetry. Since the flowfield is subsonic the farfield boundaries of the flow domain were extended approximately 15 cowl diameters away from the outer surface of the inlet. The boundary conditions at the farfield boundaries were characteristic boundary conditions that used the freestream conditions. The internal portion of the flow domain extended downstream of the diffuser exit approximately one cowl diameter to reduce the influence of the mass flow boundary condition applied on that boundary. The boundary conditions on the surfaces of the inlet and spinner were no-slip, adiabatic walls.

A structured grid was generated that contained six zones in which the grid lines matched contiguously across the zonal boundaries. The grid was clustered on the inlet and spinner surfaces to resolve the turbulent boundary layers such that the first grid point off the viscous surfaces had a $y+$ of less than 1.0. The stretching between consecutive grid points along a grid line was kept below 15 to $20 \%$. The baseline grid consisted of 439484 grid points.

Simulations were performed on the baseline grid using the Spalart-Allmaras turbulence model. Figures 7 and 8 show the comparisons between the CFD results and the experimental data for the axial static pressure distributions at 0 and 50 degrees angle-of-attack, respectively. These figures were taken directly from Ref. 9. The comparisons show good agreement, although there is considerable disagreement at the location of maximum thickness of the cowl lip, which corresponds to the lowest local static pressures.

To examine if the disagreement was due to the choice of turbulence model, simulations were performed using the Baldwin-Lomax and SST models. No significant differences in the static pressure distributions were observed between the turbulence models. Further details can be found in Ref. 9.

To examine if the disagreement was due to grid resolution, simulations were performed in which the number of grid points was doubled along the surfaces in the streamwise direction, which contained the greatest amount of curvature. No significant differences in the static pressure distributions were observed between the two grids. Further details can be found in Ref. 9. 


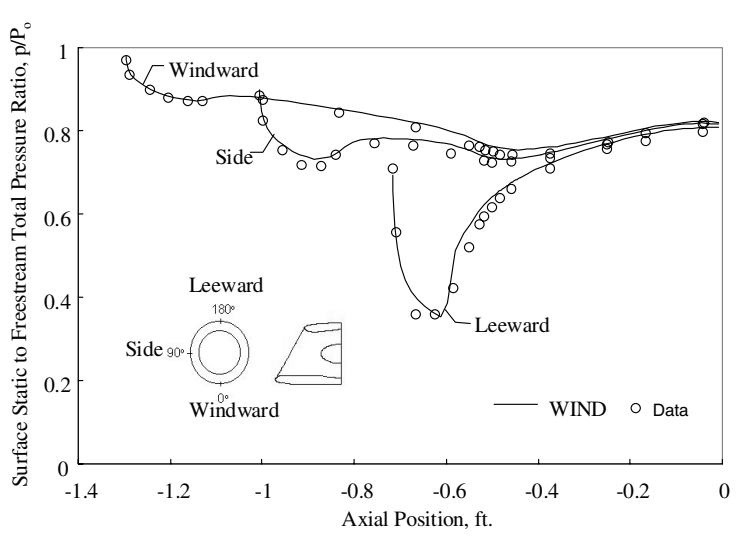

Figure 7. Comparison of the static pressure distributions in the axial direction at 0,90 , and 180 degrees at 0 degrees angle-of-attack.

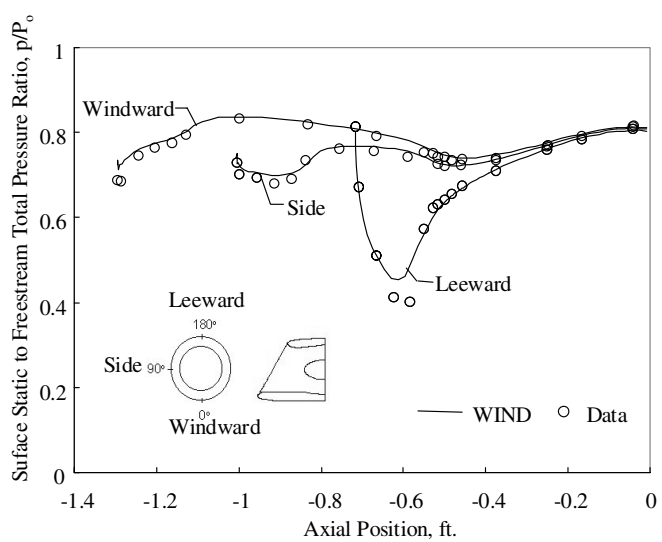

Figure 8. Comparison of the static pressure distributions in the axial direction at 0,90 , and 180 degrees at 50 degrees angle-of-attack.

The comparison of the total pressure recoveries at the diffuser exit between the experimental data and the CFD simulations with respect to the throat Mach number is shown in Fig. 9, which was taken from Ref. 9. The plots show the characteristic drop off in recovery due to higher viscous losses as the throat Mach number increases. The WIND simulations consistently under-predicted the total pressure recovery by about 0.4 percent at a throat Mach number of around 0.6 . Changing the turbulence model or grid spacing had no significant effect on the pressure recovery.

The parametric design study currently being conducted by the second author is examining the angleof-attack at which the boundary layer starts to separate from the internal surfaces of the inlet as a measure of performance of the inlet. Determining this separation angle-of-attack through the CFD simulation and comparing it to experimental data are discussed in Ref. 9. A summary of that discussion is that the validation of WIND to predict the separation angle-of-attack was not completely conclusive. The separation angle-ofattack was determined in the experiment by monitoring the static pressure on the cowl lip and total pressure at the diffuser exit. However, for CFD simulations, the separation angle-of-attack was determined by monitoring the skin friction and noting when it first became zero. The lack of experimental data that is complete and consistent with CFD simulations prevents conclusive validation of the separation angle-of-attack.

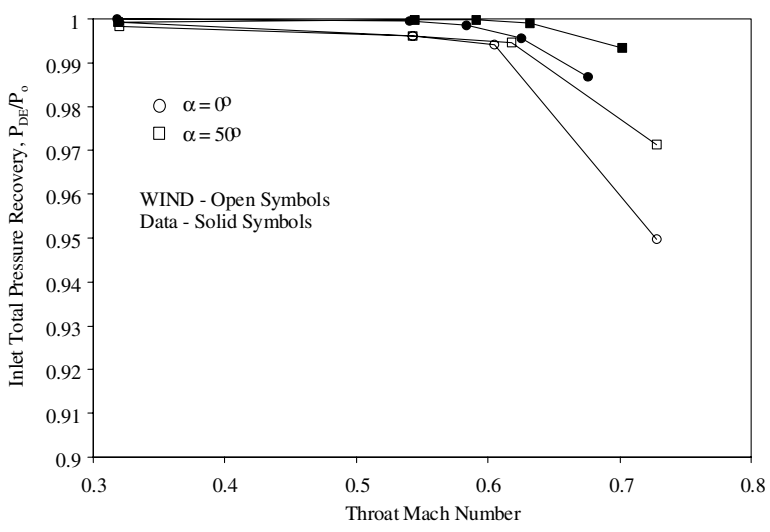

Figure 9. Comparison of total pressure recoveries.

Questions arose during the validation assessment as to whether the geometry that was simulated by the grid was the same as that tested in the wind tunnel. It was fairly certain that the CFD model matched the design specification of the scarf inlet; however, doubts existed as to whether the wind tunnel model matched those specifications. A number of CFD simulations were performed for an axisymmetric inlet that was tested in the same series as the scarf inlet. In the CFD model of the axisymmetric inlet, the leading edge radius of the CFD model was varied slightly to examine the effect of this variation on the static pressures. The details of that study are reported in Ref. 9. A summary of that discussion is that small changes in the leading edge radius had a significant effect on the static pressure distribution on the internal surfaces of the inlet near the leading edge and the location of maximum lip thickness. Furthermore, it was observed that the experimental data for the static pressure at 0 and 180-degree locations for 0 degrees angle-of-attack were not symmetric, as they should have been. All this suggested that there might have been variations in the geometry of the wind tunnel models at the leading edge and in the circumferential direction. It was indicated that the models were fabricated of fiberglass and some final "hand-finishing" was involved. This may have been enough to impact the final shape of the wind tunnel model. The wind tunnel model no longer exists to allow confirmation of this conclusion. However, this does show the impact of errors in geometry modeling. 


\section{NASA VDC Supersonic Inlet}

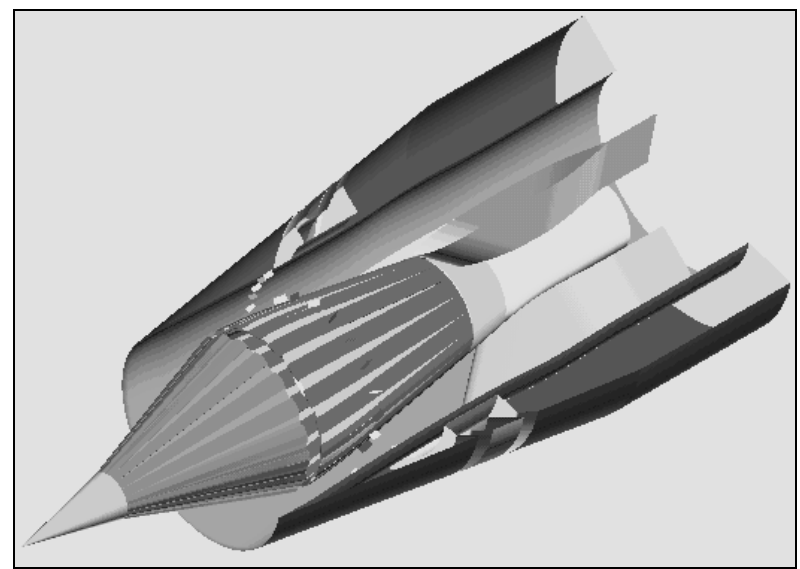

Figure 10. The geometric features of the NASA VDC mixed-compression, supersonic inlet.

The NASA Variable Diameter Centerbody (VDC) inlet was an axisymmetric, mixed-compression supersonic inlet that was studied at NASA GRC starting in the early 1970's and then tested in 1993. ${ }^{10}$ The term "mixed-compression" refers to the condition that supersonic compression occurs on the external surfaces of the centerbody and within the internal ducting of the inlet. A normal terminal shock exists downstream of the throat of the inlet to create subsonic flow for intake by a gas turbine engine. The inlet was studied as a concept to provide good pressure recovery and variable geometry for inlet operations for a vehicle capable of supersonic cruise. This inlet was of interest for a CFD validation study because it represents a class of mixedcompression, supersonic inlets that continue to be studied at NASA GRC. This validation case directly supports those studies and provides an example on the use of WIND for analyzing such inlets.

Figure 10 shows some of the main geometric features of the inlet, which includes the centerbody, cowl, and support struts. The centerbody had the feature of overlapping leaves, which allowed the centerbody to collapse in a manner similar to an umbrella. This feature allowed the cross-sectional area of the inlet to vary to adjust to inlet operating conditions. The entire centerbody mechanism could also translate forward to allow further area variation. The gap between the forward and aft portions of the centerbody served as a bleed slot. Three struts supported the centerbody. The cowl was axisymmetric and included bleed holes and passages to stabilize the shock waves and improve performance. Vortex generators were located on the centerbody and cowl surfaces downstream of the throat. The vortex generators acted to energize the surface boundary layers to reduce the likelihood of boundary layer separation in the subsonic diffuser.
The flow field about the inlet was supersonic. The case considered here involved a freestream Mach number of 2.498 with a static pressure of $0.744 \mathrm{psi}$ and a static temperature of $247.44{ }^{\circ} \mathrm{R}$. A conical shock formed at the nose of the centerbody and impinged near the cowl lip. An oblique shock was generated by the internal surface of the cowl and intersected the centerbody at its "shoulder." The turning of the shoulder nearly canceled the cowl shock. The Mach number approached 1.0 as the internal supersonic flow encountered the minimum area or throat of the inlet. A slight diffusion of the area accelerated the flow to approximately Mach 1.3 where a terminal normal shock was established to decelerate the flow to subsonic conditions while further compressing the flow. Further subsonic diffusion reduced the Mach number at the compressor face station to approximately 0.35 for ingestion by the engine. Several of these flow features are shown in the Mach number contours of Fig. 11.

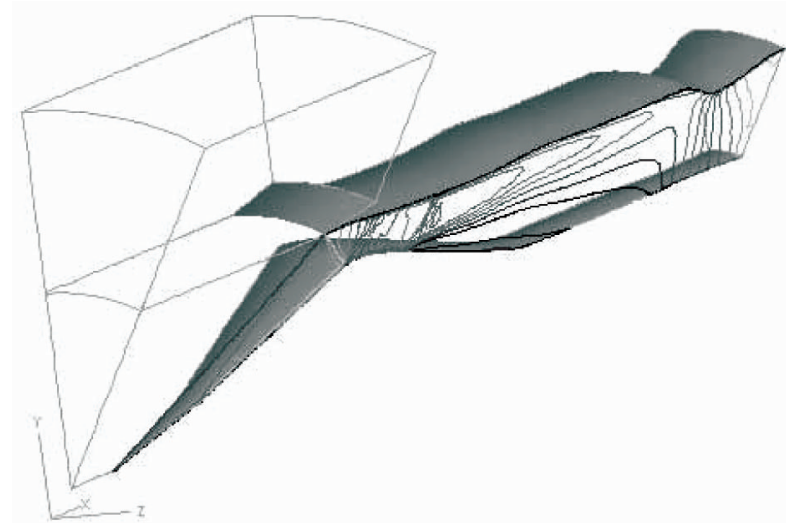

Figure 11. CFD geometry and flow domain model of the NASA VDC supersonic inlet and Mach number contours.

The inlet was tested in the 10x10 Foot Supersonic Wind Tunnel (SWT) at NASA GRC in 1993. The available experimental data consist of static pressure distributions along the centerbody and cowl and the total pressure recovery at the compressor face station. The wind tunnel tests were designed to demonstrate the inlet concept rather than provide data for CFD validation. This case can be considered a system level validation case since the test article had many of the complexities of an operational inlet and several tests were conducted with an engine attached.

The geometry modeling for the CFD simulations involved simplification of the details of the overlapping leaves and slot to represent the centerbody and cowl as continuous axisymmetric profiles. Furthermore, the vortex generators and support struts were not modeled. These simplifications introduced acknowledged physical modeling errors into the CFD simulations lessened the credibility of the validation assessment. 
The flow domain for the CFD simulations is shown in Fig. 11. Since the inlet geometry as modeled was axisymmetric, an axisymmetric flow domain was used which consisted of a 10-degree section of the inlet. Symmetry boundary conditions were applied on the sides of the 10-degree section. Since the freestream flowfield was supersonic, the inflow and farfield boundaries were placed fairly close to the exterior surfaces of the inlet, and fixed boundary conditions could be imposed. For the supersonic outflow boundaries on the exterior, extrapolation boundary condition were imposed. On the surfaces of the inlet, no-slip, adiabatic wall boundary conditions were imposed. The bleed flow through the slot and bleed holes was modeled using a bleed boundary condition in which the amount of mass flow was directly specified. The primary difficulty for a CFD simulation of a mixed-compression inlet was the establishment of the terminal shock. Here a varying area nozzle was attached to the flow domain at the compressor face station to control the amount of back-pressure at the compressor face station and position the terminal shock.

A planar, structured grid was generated with two zones-one placed along the centerbody for the internal flow and a second placed along the cowl for the external flow. The three-dimensional grid was obtained by extruding the planar grid circumferentially over the 10-degree section of the flow domain. The grid was clustered near the surfaces of the inlet to resolve the turbulent boundary layers such that the first grid point off the wall results in a $y+$ value of less than 1.0.

The CFD simulations were performed at zero angleof-attack with a bleed mass flow of $2.575 \%$ of the nominal captured mass flow imposed at the centerbody bleed slot. The initial flow field for the simulation was the freestream conditions. The supercritical flow was first developed in which there was supersonic flow through the inlet. The nozzle area was then reduced until subsonic flow and a normal terminal shock developed in the diffuser. The nozzle area was then gradually reduced until the static pressure at the compressor face station matched that of the experiment.

The baseline simulation was performed using the Spalart-Allmaras turbulence model. The comparison of the static pressures along the centerbody and cowl as computed by WIND with those of the experiment is shown in Fig. 12. Estimates of the experimental error within the static pressures were not available. Overall, the comparisons are good. Figure 12(a) shows the profile of the inlet for reference. WIND accurately predicts the increase in pressure across the change in conical sections of the forward portion of the centerbody. The sharp rise in static pressure near the slot and shoulder is due to the shock generated by the cowl lip that intersects the centerbody just upstream of the slot. The bleed that is extracted at the slot prevents excessive boundary layer separation which would grow and result in the unstart of the inlet. Downstream of the shoulder, the static pressure drops and then rises sharply across the terminal shock. The comparison with the experimental data suffers at this point. It is believed that the differences between the experimental data and the CFD results at this point are due to the lack of the modeling of the vortex generators in the WIND simulation. A large region of boundary layer separation existed in the CFD simulation at this point, which was probably not present in the experiment as indicated by the lower static pressures.

Figure 12(b) shows the comparison of static pressures on the interior cowl surface. The axis has a smaller range than Fig. 12(a), and so, the differences show up more; however, the comparisons are good.

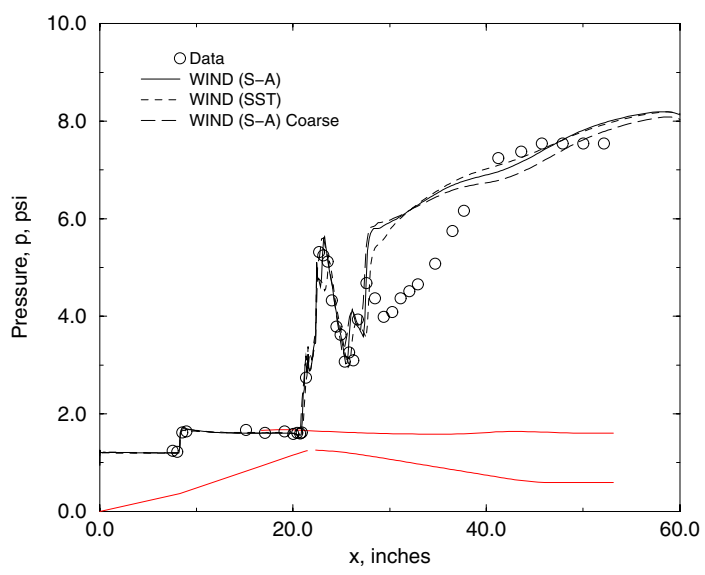

(a) Centerbody.

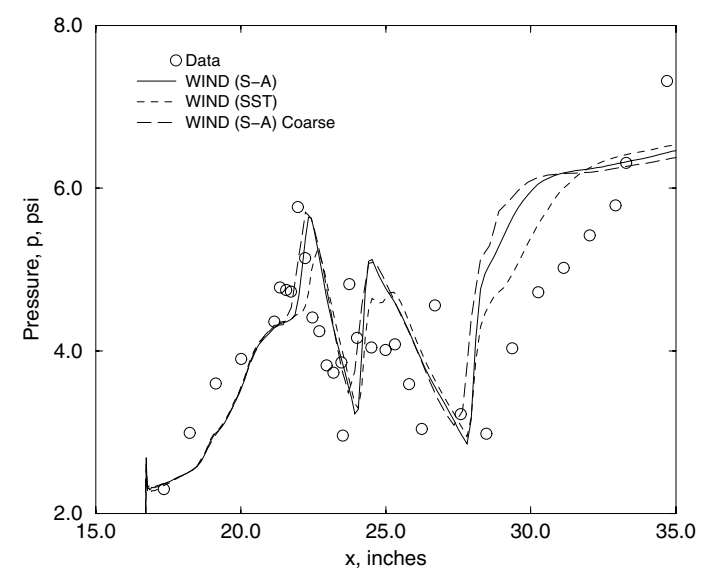

(b) Cowl.

Figure 12. Comparison of static pressures computed by WIND with those of experiment for the NASA VDC supersonic inlet. 
Typically the static pressure data are the easiest to match by CFD. In supersonic, internal, turbulent flows, the static pressure is significantly affected by shock location and shock / boundary layer interactions. Thus, comparisons with static pressures are more meaningful than for subsonic inlet experiments.

Figure 12 shows the effects of grid refinement on the pressure distributions by comparing the results on the baseline and coarse grids. The coarse grid contained every other grid point of the baseline grid in the streamwise and radial directions. Some of the peaks are not resolved as well, but the coarse grid solution was essentially the same as the baseline grid solution. This provided confidence that the baseline grid was of sufficient resolution.

Figure 12 includes the static pressure distribution obtained using the SST turbulence model. The static pressures for the SST model follow the behavior of the Spalart-Allmaras model. The cowl shock and terminal shock are slightly further downstream than for the Spalart-Allmaras model. Examination of the boundary layer on the centerbody indicated that the boundary layer thickness for the Spalart-Allmaras model was greater than that for the SST model. This affected the blockage through the inlet. The thicker boundary layers tended to push the terminal shock forward slightly. Simulations using the Baldwin-Lomax turbulence model over-predicted the extent of the boundary layer separation in the subsonic diffuser and would not yield a reasonably steady-state solution.

The experiment indicated the total pressure recovery as computed on a 40-probe, area-weighted rake to be 0.699. The baseline CFD simulations using the SpalartAllmaras model indicated a total pressure recovery of 0.726 on the fine grid and a recovery of 0.719 on the coarse grid. The CFD simulation using the SST model indicated a recovery of 0.729 . These represent an approximately $4.0 \%$ difference from the experimental value. The higher pressure recoveries for the CFD simulations are questionable considering that the vortex generators were not modeled and large regions of boundary layer separations were observed in the simulations. One would expect the experimental pressure recovery to be higher than those of the CFD simulations. The pressure recoveries from the CFD simulations were computed using a mass-weighted average on the CFD grid at the compressor face station rather than interpolating the $\mathrm{CFD}$ results to the rake positions. The differences in these approaches to computing the recovery from the CFD results can be evaluated; however, past comparisons indicated a small amount of difference. It was assumed that such a difference would be small for these simulations and given the simplifying approximations of the geometry modeling, probably not meaningful to examine.

\section{$\underline{\text { RBCC Strut-Jet Hypersonic Inlet }}$}

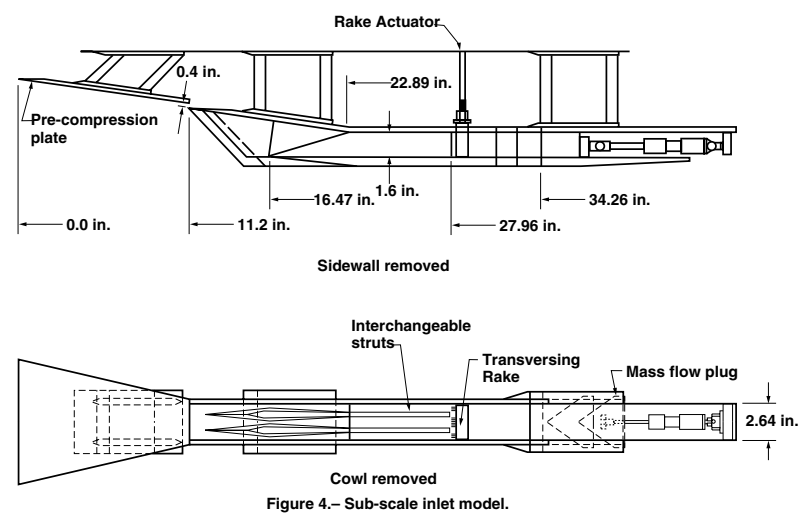

Figure 13. The geometry of the RBCC Strut-Jet hypersonic inlet.

The Strut-Jet hypersonic inlet was a concept studied at NASA GRC as an inlet for a rocket-based combinedcycle (RBCC) engine. " The term "combined cycle" refers to the combination of rocket and ramjet engine cycles that allow such an engine to operate from static conditions to beyond Mach 8. This inlet was of interest for a CFD validation study because it represents a class of hypersonic inlets that continue to be studied at NASA GRC. This case supports those studies.

Figure 13 shows a diagram of the geometry of the Strut-Jet inlet. The inlet had a rectangular cross-section with two struts that divided the inlet flow into three separate flow paths that combined again at the base of the struts. The inlet was symmetric with respect to a plane down the center of the center flow path. Rocket engines were located within the base of each strut.

The flowfield was hypersonic with a freestream Mach number of 6.0. A pre-compression plate ahead of the inlet generated an oblique shock that entered the inlet and reflected off the cowl. Oblique shocks were also generated by the leading edges of the struts and entered the inlet. In operations as a ramjet, the subsonic combustion would occur downstream of the struts. The case considered here is that for supercritical flow in which there is supersonic flow throughout the inlet.

The experiment was conducted in the NASA GRC 1x1 Foot SWT. ${ }^{11}$ The experimental data available for comparison consist of static pressure distributions along the centerline of the center flow passage on the cowl and body, static pressure distributions on the inner surfaces of the left and right sidewalls, total pressure maps at the end of the isolator, and exit total pressure recovery and kinetic energy efficiency. This case can be considered a subsystem validation case since the test article was a sub-scale model of the actual inlet and considered only the aerodynamics of the inlet rather than the combined inlet and ramjet operation. 


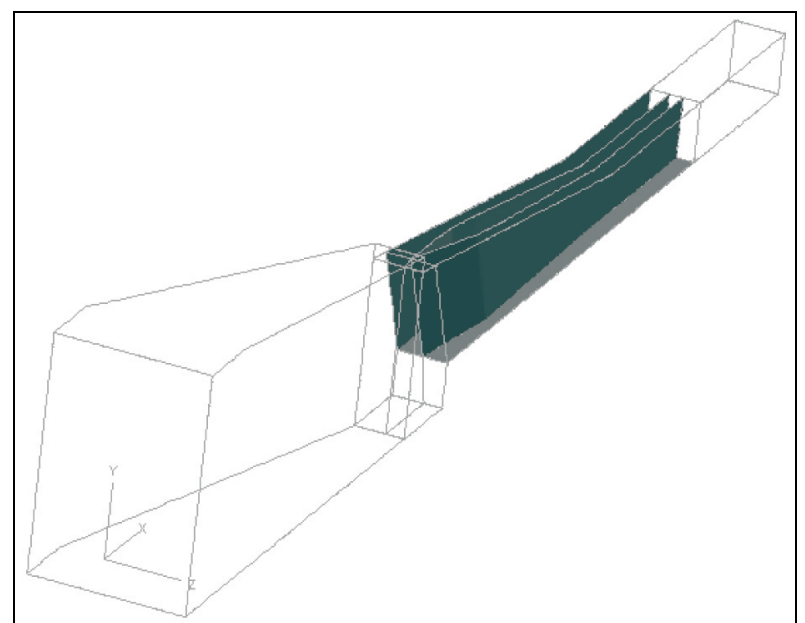

Figure 14. Flow domain and surfaces of RBCC Strut-Jet inlet.

Figure 14 shows the flow domain, which used symmetry to allow the modeling of only half of the inlet. The boundary conditions were fixed at inflow boundaries and extrapolated at the outflow boundaries. No-slip, adiabatic wall boundary conditions were imposed on the pre-compression plate and inlet surfaces. The initial flow field was set to freestream.

The CFD simulations used the same grid as the simulations of Ref. 12, which used the NPARC code. The grid consisted of 6 zones with the grid clustered near the inlet surfaces with a normal wall spacing of approximately $4.0 \mathrm{E}-05$. The total size of the grid was 1381946 grid points. The CFD simulations were performed with the assumption of completely turbulent flow using the Spalart-Allmaras and SST models.

Figure 15 show the comparison of the experimental data and CFD results for the static pressures on the body and cowl surfaces. The experimental error in measuring the static pressure was reported to be 0.02 psi, which was too small to be plotted as error bars. The comparisons between the experimental data and CFD results are good. Both turbulence models gave essentially the same results, but the SpalartAllmaras model tended to indicate higher peaks in the static pressure. Both models seemed to place the shock locations slightly downstream of the experimental data. Also plotted in Fig. 15 are the results from the CFD study of Ref. 12 that used the NPARC CFD code with the Chien $\mathrm{k}-\varepsilon$ turbulence model. The NPARC results indicate a large spike in the pressures on the cowl at approximately $\mathrm{x}=17$ inches that is not indicated in the WIND simulations. This may be due to the use of an upwind TVD limiter in WIND, whereas the NPARC code used a central-difference formulation with artificial dissipation. Elsewhere along the distribution, the NPARC results compare well with the experimental data and perhaps compare better than the WIND results.

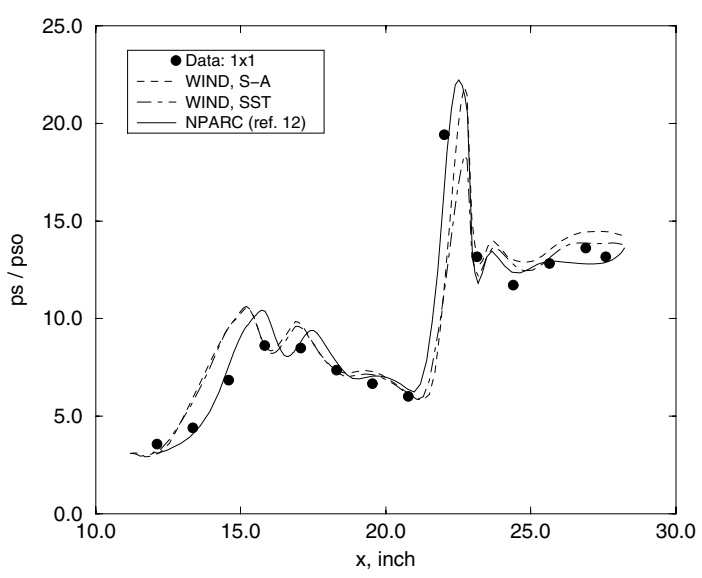

(a) Body

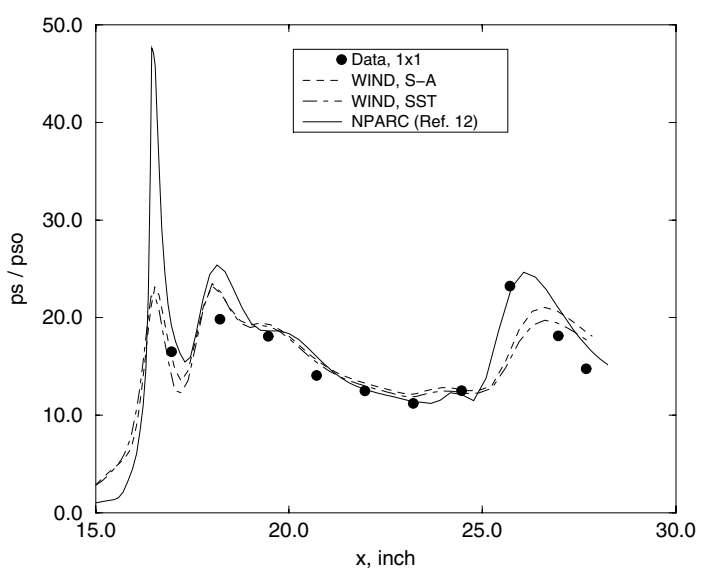

(b) Cowl

Figure 15. Comparison of the static pressure distributions along the body and cowl of the RBCC strut-jet hypersonic inlet.

The comparison of the total pressure recoveries and kinetic energy efficiencies is presented in Table 1. Both CFD simulations over-predict the performance. The kinetic energy efficiencies of the CFD simulations were computed using the mass-averaged total pressures and Mach numbers of the center and side passages. The percent difference of the CFD values to the experimental values is indicated in parenthesis. The large differences in total pressure recovery become smaller differences in kinetic energy efficiency.

Table 1. Performance of the Strut-Jet inlet.

\begin{tabular}{|l|l|l|}
\hline & \multicolumn{1}{|c|}{$\boldsymbol{\eta}_{\mathbf{p t}}$} & \multicolumn{1}{c|}{$\boldsymbol{\eta}_{\text {KE }}$} \\
\hline Data, 1x1 & 0.30 & 0.943 \\
\hline WIND, S-A & $0.3186(6.2 \%)$ & $0.9463(0.35 \%)$ \\
\hline WIND, SST & $0.3402(13.4 \%)$ & $0.9499(0.73 \%)$ \\
\hline
\end{tabular}




\section{GTX Rig 2.1 Hypersonic Inlet}

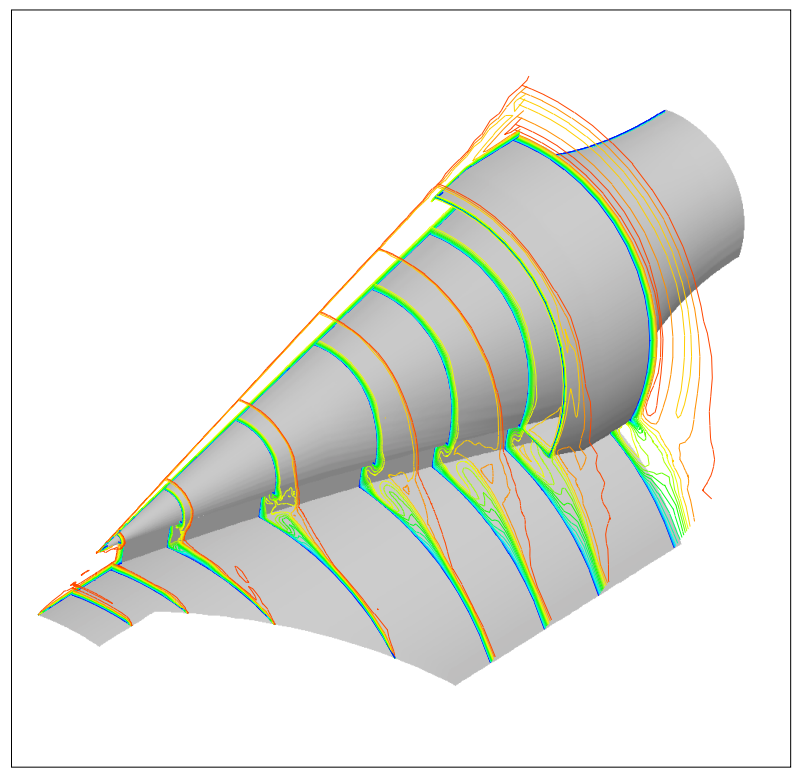

Figure 16. Geometry and Mach number contours for the GTX Rig 2.1 sub-scale hypersonic inlet.

The GTX Rig 2.1 hypersonic inlet was a sub-scale inlet model studied at NASA GRC as part of a current research program to develop technologies for a rocketbased combined-cycle (RBCC) engine. ${ }^{13}$ This inlet was of interest for a CFD validation study because the study supported the use of the WIND code for the aerodynamic analysis of the inlets for the GTX program.

Figure 16 shows the geometry of the inlet. Only half of the inlet is shown since it is symmetric about the center plane. The inlet was a 220-degree section of an axisymmetric inlet and consisted of a conical centerbody and an axisymmetric cowl. The inlet was mounted on a pylon that set the inlet above the boundary layer generated on the vehicle body. The vehicle has three such inlets mounted about the circumference of the body. The experiment used a body simulator fairing that started just ahead of the nose of the centerbody. The centerbody was translated as to provide a geometric contraction ratio of 7.14. The half-angle of the conical centerbody was 12 degrees.

The experiment was conducted in the NASA GRC 1x1 Foot SWT. ${ }^{13}$ The inflow conditions were a Mach number of 6.02, a total pressure of $146 \mathrm{psi}$, and a total temperature of $811{ }^{\circ} \mathrm{R}$. Figure 16 shows the Mach number contours. The center-body generated a conical shock that passed just outside of the cowl lip. The conical shock interacted with the turbulent boundary layer on the body fairing to generate local disturbances of the boundary layer on the body.

The experimental data consist of streamwise static pressure distributions along the centerbody and cowl at various circumferential stations. This case can be

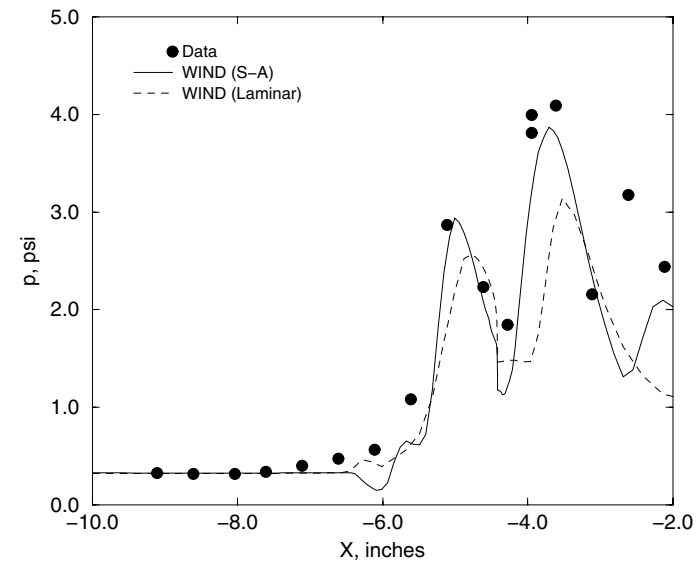

(a) centerbody

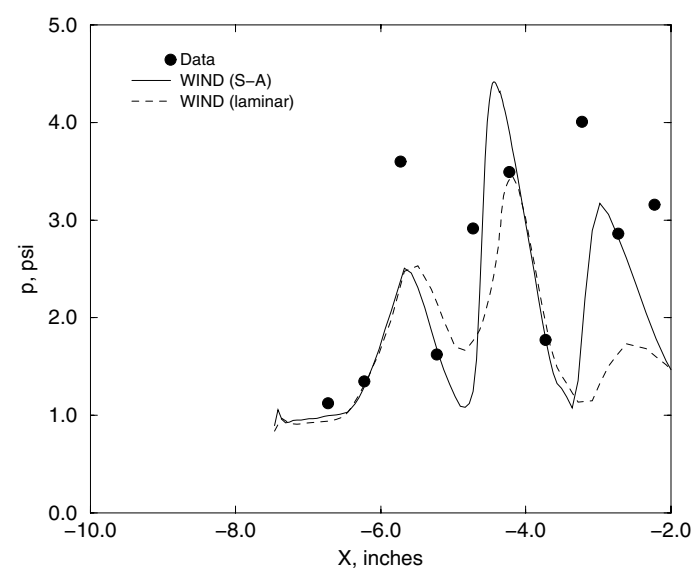

(b) cowl

Figure 17. Comparison of the static pressures at the centerline plane along the centerbody and cowl for the GTX Rig 2.1 hypersonic inlet.

considered a subsystem validation case since the test article was a sub-scale model of the actual inlet and only examined the aerodynamics of the inlet.

The baseline grid consisted of 19 zones with a total of 2469391 grid points. The grid points were clustered near the surfaces to resolve the turbulent boundary layers with $\mathrm{y}+$ values less than 1.0 for the first grid points off the surfaces. A coarse grid was extracted from the baseline grid by removing every other grid point in each coordinate direction.

The WIND simulations used the tunnel conditions as its initial solution. The inlet was simulated in supercritical mode in which the core flow remains supersonic throughout the inlet. The baseline simulation used the Spalart-Allmaras turbulence model. The total pressure recovery from the CFD results was 
0.41998 on the baseline grid and 0.41032 on the coarse grid, which was about a $2.3 \%$ difference and assured that the baseline grid provided acceptable resolution.

Figure 17 shows the comparison between the CFD results and the experimental data for the static pressure distributions along the centerbody and cowl at the centerline of the inlet. The experimental error in measuring the static pressure was reported to be $0.02 \mathrm{psi}$, which was too small to be plotted as error bars. The "peaks" and "valleys" indicate the presence of oblique shocks bouncing through the inlet near the throat. The results of the baseline simulation compare well with the experimental data; however, a noticeable "dip" in pressure occurs on the centerbody at approximately $\mathrm{x}=-6.0$ inches, which is not indicated in the experimental data. This is the location at which the cowl shock impinges onto the centerbody. The experimental data suggests that there is a small separation region. It was suggested that the flow was not fully turbulent as assumed. Simulations were then performed assuming laminar flow. Figure 17 shows those distributions of the static pressure. The laminar simulation indicated a small separation region and higher values of static pressures at the location of the "dip." This suggested that the flow on the centerbody was perhaps a mix of laminar and turbulent flow, which was difficult to simulate with CFD methods and confounds a conclusive validation assessment.

\section{Summary and Conclusions}

A series of cases has been presented for the validation assessment of the WIND code that increases the confidence in the use of WIND for the CFD analysis of aerodynamic of aircraft inlets. The assessment mainly examined the prediction of static pressure distributions and pressure recovery. The use of WIND to predict such things as inlet drag requires further validation assessment. It should also be understood that validation is an on-going process and the use of any CFD code relies on a basic understanding of the flow problem and of the flow physics involved.

\section{$\underline{\text { References }}$}

1. AIAA, "Guide for the Verification and Validation of Computational Fluid Dynamics Simulations," AIAA G-077-1998, 1998.

2. Bush, R.H., G.D. Power, and C.E. Towne, "WIND: The Production Flow Solver of the NPARC Alliance," AIAA Paper 0935, January 1998.
3. WIND CFD Code web site: http://www.grc.nasa.gov/www/winddocs.

4. Slater, J.W., "Verification Assessment of Flow Boundary Conditions for CFD Analysis of Supersonic Inlet Flows," AIAA-2001-3882, July 2001.

5. Roache, P.J., Verification and Validation in Computational Science and Engineering, Hermosa Publishers, Albuquerque, New Mexico, 1998.

6. Stanitz, J.D., W.M. Osborn, and J. Mizisin, “An Experimental Investigation of Secondary Flow in an Accelerating, Rectangular Elbow," NACA TN3015, October 1953.

7. Cavicchi, R.H., "A Three-Dimensional CFD Investigation of Secondary Flow in an Accelerating, 90 $0^{\circ}$ Elbow," NASA/TM-2001211219. December 2001.

8. Abbott, J.M, "Aerodynamic Performance of Scarf Inlets,” NASA TM-79055, January 1979.

9. Abbott, J.M. and J.W. Slater, "Computational Study of the Aerodynamic Performance of ThreeDimensional Subsonic Inlets," AIAA-2001-3886, July 2001.

10. Wasserbauer, J.F., R.J. Shaw, and H.E. Neumann, "Design of a Very-Low-Bleed Mach 2.5 MixedCompression Inlet with 45 Percent Internal Contraction," NASA TM X-3135, 1974.

11. Fernandez, R., C.J. Trefny, S.R. Thomas, and M.J. Bulman, "Parametric Data from a Wind Tunnel Test of a Rocket-Based Combined-Cycle Engine Inlet," NASA/TM-2001-107181, November 2001 .

12. DeBonis, J.R. and S. Yungster, "Rocket-Based Combined Cycle Engine Technology Development - Inlet CFD Validation and Application," NASA TM-107274 (AIAA-96-3145), July 1996.

13. DeBonis, J.R., C.J. Trefny, and C.J. Steffen, Jr., "Inlet Development for a Rocket Based Combined Cycle, Single Stage to Orbit Vehicle Using Computational Fluid Dynamics," AIAA-99-2239, June 1999.

14. DeBonis, J.R. and C.J. Trefny, "Supersonic Wind Tunnel Tests of a Half-Axisymmetric $12^{\circ}$-Spike Inlet to a Rocket-Based Combined-Cycle Propulsion System," JANNAF, November 2000.

15. NPARC Verification and Validation web site: http://www.grc.nasa.gov/www/wind/valid. 


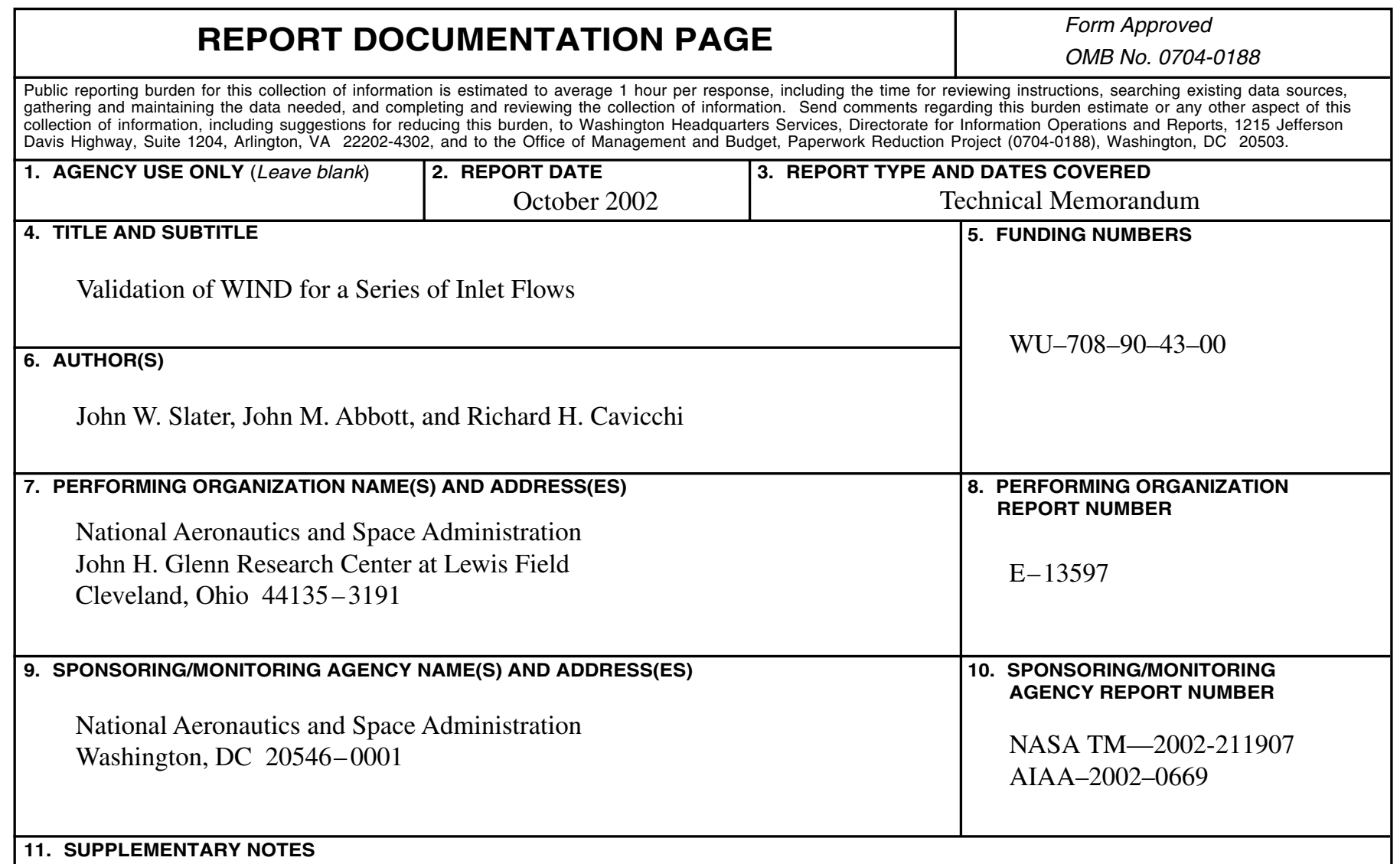

Prepared for the 40th Aerospace Sciences Meeting and Exhibit sponsored by the American Institute of Aeronautics and Astronautics, Reno, Nevada, January 14-17, 2002. Responsible person, John W. Slater, organization code 5850,

216-433-8513.

12a. DISTRIBUTION/AVAILABILITY STATEMENT

12b. DISTRIBUTION CODE

Unclassified - Unlimited

Subject Categories: 02 and 07

Distribution: Nonstandard

Available electronically at http://gltrs.grc.nasa.gov

This publication is available from the NASA Center for AeroSpace Information, 301-621-0390.

13. ABSTRACT (Maximum 200 words)

Validation assessments compare WIND CFD simulations to experimental data for a series of inlet flows ranging in Mach number from low subsonic to hypersonic. The validation procedures follow the guidelines of the AIAA. The WIND code performs well in matching the available experimental data. The assessments demonstrate the use of WIND and provide confidence in its use for the analysis of aircraft inlets.

\begin{tabular}{|c|c|c|}
\hline \multicolumn{3}{|c|}{$\begin{array}{l}\text { Computational fluid dynamics; Inlet flow; Subsonic flow; Supersonic flow; } \\
\text { Hypersonic flow }\end{array}$} \\
\hline $\begin{array}{l}\text { 17. SECURITY CLASSIFICATION } \\
\text { OF REPORT }\end{array}$ & $\begin{array}{l}\text { 18. SECURITY CLASSIFICATION } \\
\text { OF THIS PAGE }\end{array}$ & $\begin{array}{l}\text { 19. SECURITY CLASSIFICATION } \\
\text { OF ABSTRACT }\end{array}$ \\
\hline Unclassified & Unclassified & Unclassified \\
\hline
\end{tabular}

